\title{
Directly Phase-Modulated Light Source
}

\author{
Z. L. Yuan, ${ }^{1, *}$ B. Fröhlich, ${ }^{1}$ M. Lucamarini, ${ }^{1}$ G. L. Roberts, ${ }^{1,2}$ J. F. Dynes, ${ }^{1}$ and A. J. Shields ${ }^{1}$ \\ ${ }^{1}$ Toshiba Research Europe Limited, 208 Cambridge Science Park, \\ Milton Road, Cambridge, CB4 OGZ, United Kingdom \\ ${ }^{2}$ Cambridge University Engineering Department, $9 \mathrm{~J}$ J Thomson Avenue, \\ Cambridge, CB3 OFA, United Kingdom
}

(Received 15 April 2016; revised manuscript received 18 August 2016; published 20 September 2016)

The art of imparting information onto a light wave by optical signal modulation is fundamental to all forms of optical communication. Among many schemes, direct modulation of laser diodes stands out as a simple, robust, and cost-effective method. However, the simultaneous changes in intensity, frequency, and phase have prevented its application in the field of secure quantum communication. Here, we propose and experimentally demonstrate a directly phase-modulated light source which overcomes the main disadvantages associated with direct modulation and is suitable for diverse applications such as coherent communications and quantum cryptography. The source separates the tasks of phase preparation and pulse generation between a pair of semiconductor lasers leading to very pure phase states. Moreover, the cavity-enhanced electro-optic effect enables the first example of subvolt half-wave phase modulation at high signal rates. The source is compact, stable, and versatile, and we show its potential to become the standard transmitter for future quantum communication networks based on attenuated laser pulses.

DOI: 10.1103/PhysRevX.6.031044

Subject Areas: Optics, Photonics, Quantum Information

\section{INTRODUCTION}

Phase modulation is an important encoding format for optical communications [1], and it forms the basic building block for other signal formats such as amplitude, polarization [2], and quadrature-amplitude modulation [3]. Its primary enabling technology is external modulation, where the phase is changed by acting on the electric field-dependent refractive index of certain electro-optic materials [4]. Conventional phase modulators based on $\mathrm{LiNbO}_{3}$ crystals [5] or semiconductor waveguides [6] require driving voltages beyond the reach of complementary metal-oxide semiconductor (CMOS) logic, necessitating the use of amplifiers which add to the system cost and complexity. Their prospects for a subvolt half-wave voltage $\left(V_{\pi}\right)$ suitable for direct CMOS driving are limited. Substantially increasing the device length is both undesirable and difficult. $\mathrm{LiNbO}_{3}$ phase modulators already possess a length of several centimeters, while semiconductor devices suffer from impedance matching problems [7] and considerable insertion losses at longer lengths. As an alternative, organic dielectric materials show promising electro-optic coefficients [8] but as of yet have not resulted in a subvolt phase modulator [9].

Direct modulation of laser diodes is attractive because no external modulator is necessary, and it can be achieved with

\footnotetext{
* Corresponding author. zhiliang.yuan@crl.toshiba.co.uk
}

Published by the American Physical Society under the terms of the Creative Commons Attribution 3.0 License. Further distribution of this work must maintain attribution to the author(s) and the published article's title, journal citation, and DOI. low driving voltages $[10,11]$. However, spurious effects such as frequency chirp, large intensity fluctuations, and uncontrolled phase evolution limit its applicability. To date, it has been used primarily for on-off keying in classical communications with restricted data bandwidth and communication distances [3]. Although it provides phase modulation, the dominant amplitude variation makes it nonideal for state-of-the-art coherent communication systems [12]. In challenging applications like quantum cryptography [13-15], direct modulation fails altogether, as the unintentional changes in other degrees of freedom cause a side-channel information leakage [16].

Here, we introduce a novel concept for direct phase modulation in a light source, which combines the phase purity typical of external phase modulators with an exceptionally low driving voltage, entailed by the direct modulation of the laser gain. It employs only laser diodes as active components and removes the need for external modulators, making it attractive for many applications, including quantum cryptography, as we demonstrate in this work.

\section{DIRECT PHASE MODULATION SCHEME}

The central idea of the scheme is based on optical injection [17-19], which allows us to assign to different lasers the tasks of pulse generation and phase preparation. As schematically depicted in Fig. 1(a), a first laser diode is connected to a second laser diode via an optical circulator. The phase-preparation laser is directly modulated to produce long pulses from quasi-steady-state emission. Each of these pulses coherently seeds a block of two or more secondary, short optical pulses emitted by gain switching 
the pulse-generation laser. The relative phase between the secondary pulses depends on the phase evolution of the primary pulses and can be set to an arbitrary value by directly modulating the driving current applied to the phase-preparation laser. For example, a relative phase $\phi_{1}$ between two secondary pulses can be obtained by introducing a small perturbation in the driving signal of the phase-preparation laser [see Fig. 1(a)]. Similarly, the relative phases between three secondary pulses can be set to $\phi_{1}$ and $\phi_{2}$ by adding two small perturbations to the driving signal of the phase-preparation laser. In principle, such perturbations in the driving signal would cause detrimental fluctuations in the intensity and frequency of the primary pulses. However, we avoid these side effects by switching the pulse-generation laser's gain off in correspondence with the perturbation signals. Effectively, the pulse-generation laser also acts as a filter rejecting residual modulations.

An intuitive picture helps us to understand how an optical phase is set by perturbing the driving signal applied to the phase-preparation laser, Figs. 1(b) and 1(c). Consider a continuous wave laser above threshold emitting at the central frequency $\nu_{0}$. When a small perturbation is applied to the driving signal, the optical frequency shifts by an amount $\Delta \nu$, changing the course of the phase evolution. When the perturbation is switched off, the frequency is restored to the initial value $\nu_{0}$. This perturbation will create a phase difference

$$
\Delta \phi=2 \pi \Delta \nu t_{m},
$$

where $t_{m}$ is the duration of the perturbation. Through optical injection, this phase difference is transferred onto a pair of secondary pulses emitted by the pulse-generation laser [Fig. 1(d)].

The perturbation signal here is an electrical voltage modulation applied to the phase-preparation laser. The optical frequency change arises from the effect of the carrier density on the refractive index in the laser active medium [20]. The laser cavity confinement allows the light field to oscillate back and forth within the cavity and experience the refractive-index change for the entire duration of the perturbation. The enhancement due to the laser cavity allows us to keep the phase modulation half-wave voltage below $1 \mathrm{~V}$, as we demonstrate later. This cavity feature is absent in conventional phase modulators $[5,6]$, where the light makes just a single pass across the electro-optic medium, thus limiting the interaction distance to the device length.

\section{CHARACTERIZATION}

In this section, we perform a series of experiments aimed at characterizing the directly phase-modulated light source. An asymmetric Mach-Zehnder interferometer [Fig. 2(a)] is used to measure the relative phases of a train of short pulses emitted at 500-ps intervals from the pulse-generation laser. This interval is sufficiently long to extinguish the field within the pulse-generation laser between two consecutive pulses, such that lasing can be triggered by either spontaneous emission [21] (with random phase) or the phase-preparation laser (with defined phase). Figure 2(a) (right panel) compares the case where the pulse-generation laser is seeded
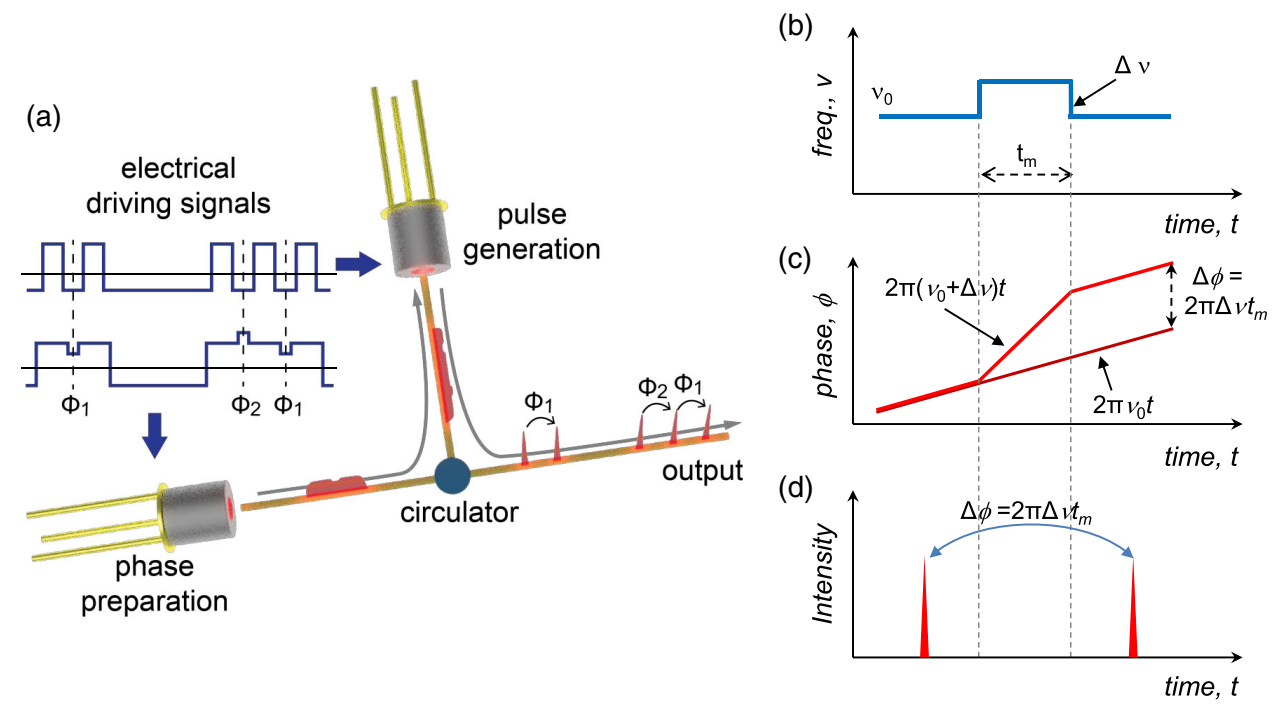

FIG. 1. Directly phase-modulated light source. (a) The source consists of a pair of semiconductor laser diodes connected via an optical circulator. We refer to these laser diodes as the phase-preparation and pulse-generation lasers. The phase-preparation laser is biased to produce nanosecond scale, quasi-steady-state optical pulses with shallow intensity modulation, which also modifies the optical phase. The gain-switched pulse-generation laser emits short optical pulses which inherit the optical phase prepared by the phase-preparation laser. The duration of each phase-preparation laser pulse can be varied to seed a pulse train of different lengths. (b) The optical frequency of the phase-preparation laser under a small perturbation of duration $t_{m}$. (c) Optical phase trajectories with and without the perturbation of the phase-preparation laser. (d) The phase $\Delta \phi$ transferred to the relative phase between pulses emitted by the pulse-generation laser. 
(a)
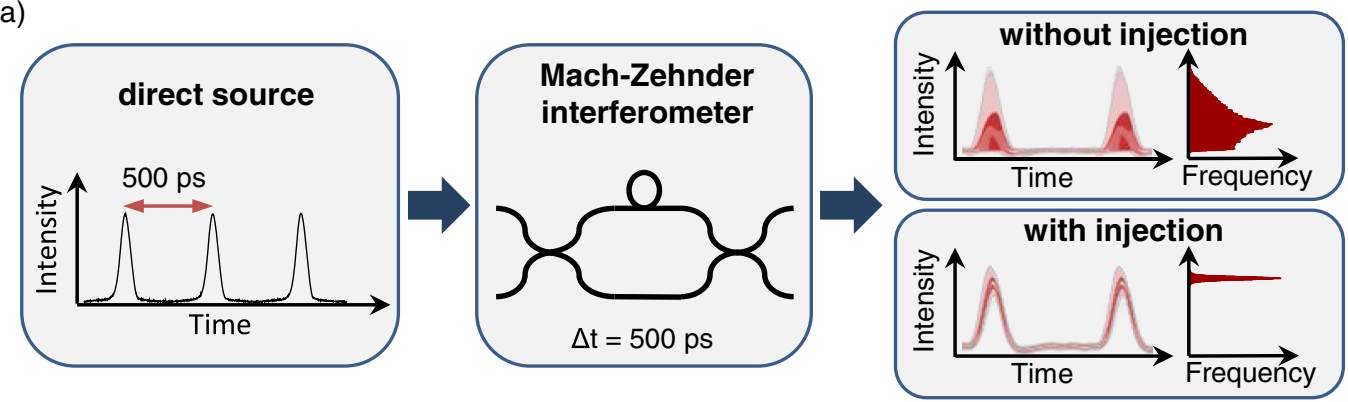

(b)

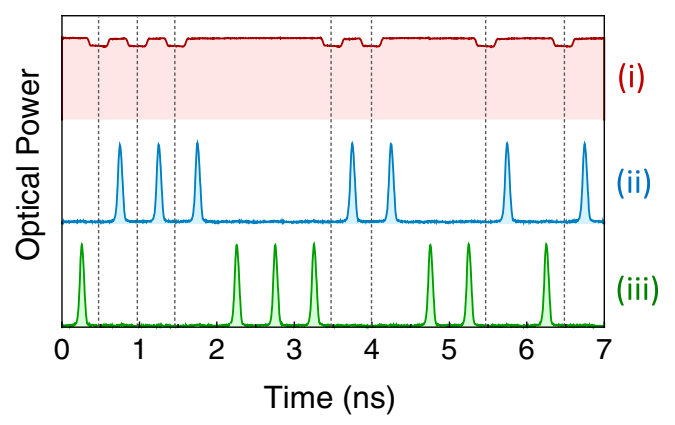

(c)

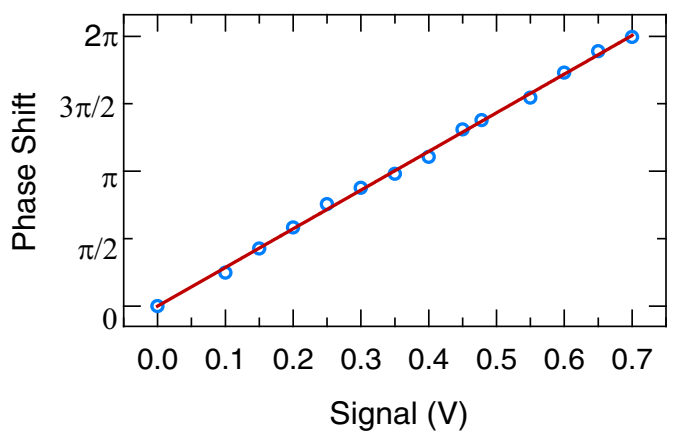

FIG. 2. Coherence seeding and phase modulation. (a) Experimental setup for evaluation of the relative phase between adjacent short pulses from the directly phase-modulated light source: The source transmits a pulse train of 500-ps intervals, and an asymmetric MachZehnder interferometer (MZI) with a matching delay is used to measure the interference. Panels on the right show color-coded density plots of the measured waveforms and corresponding histograms of peak intensities for the cases with and without optical injection, respectively. (b) Demonstration of direct phase modulation: (i) Output intensity profile of the phase-preparation laser with shallow intensity modulation; (ii, iii) complementary intensity profiles recorded from the output of the MZI. (c) Phase shift as a function of the electrical signal amplitude applied to the phase-preparation laser. In (b) and (c), the modulation signal has a duration of 250 ps. An optical injection power of $50 \mu \mathrm{W}$ is used.

with light from the phase-preparation laser to the case where it is unseeded. The unseeded case produces output waveforms of random intensities, while an injection of continuous-wave light leads to a fixed phase difference and a stable output intensity. The fidelity of the phase transfer between the laser diodes is evaluated by the interference visibility of the short pulses, which is found to grow with the injection strength and saturates at $99.06 \%$ with a modest injection power of $50 \mu \mathrm{W}$. The saturated visibility is limited by the coherence of the phase-preparation laser.

We now demonstrate phase modulation by applying a small perturbation pattern to the electrical drive signal of the phase-preparation laser, which produces a shallow intensity variation, row (i) in Fig. 2(b). A key point to note here is that the perturbation does not disrupt the phase continuity but only changes the phase evolution rate. We temporally align each modulated signal to the interval of a pair of output pulses whose relative phase is to be modified. This arrangement ensures the indistinguishability among the output pulses because they are seeded by the unmodulated part of the injected light. The successful transfer of the electrical drive pattern to the output phase is confirmed by the resulting interference waveforms, which reproduce the modulation pattern: rows (ii) and (iii) in Fig. 2(b). Here, each intensity perturbation has been calibrated to impart a $\pi$ phase shift to two consecutive output pulses. Whenever a perturbation is present in row (i) of Fig. 2(b), a pulse is also present in the $\pi$-phase output of the interferometer, row (ii) of Fig. 2(b). When there is no perturbation, the phase difference between consecutive pulses amounts to zero, and a pulse appears in the zero-phase output of the interferometer instead [row (iii) of Fig. 2(b)].

Figure 2(c) shows the phase shift measured as a function of the modulation voltage applied to the phase-preparation laser. The phase shift can be either positive or negative, and is approximately linear with the signal amplitude. The halfwave voltage amounts to only $0.35 \mathrm{~V}$. We ascribe this low $V_{\pi}$ to the aforementioned cavity enhancement, which enables an effective interaction distance of $25 \mathrm{~mm}$ within the active medium, even though its length is only about $100 \mu \mathrm{m}$. As the $V_{\pi}$ is sufficiently low to be driven directly by CMOS logic, we expect that this breakthrough will play a crucial role in reducing the complexity, as well as the energy consumption and the physical footprint, of a phase-modulated light source.

\section{APPLICATIONS TO QUANTUM COMMUNICATION}

To highlight the versatility of the source and to demonstrate its practicality, we apply it to a quantum 
communication scheme, more specifically, to various quantum key distribution (QKD) protocols. In $\mathrm{QKD}$, the most common way to transmit quantum information is as an optical phase delay between weak coherent pulses, as this has been shown to be robust even for transmission over installed fiber networks [22-25]. For the well-known BB84 protocol [13], these pulses have to fulfill one fundamental security requirement; i.e., the global phase of each encoded pulse pair has to be completely random [26]. This requirement has led to the usual implementation by passing a laser pulse through an asymmetric MachZehnder interferometer [27] because gain-switched laser pulses have been shown to feature random phases [21]. However, such implementation requires several discrete free space or fiber optical devices and components [28,29] and results in a structurally cumbersome transmitter that needs active stabilization [30] in addition to requiring powerful driving electronics for phase modulation. We note that such a BB84 encoder is incompatible with other QKD protocols, such as differential-phase-shift (DPS) [31] and coherent-one-way (COW) [32].

We introduce phase randomization by modifying the driving signal to the phase-preparation laser. The driving signal is set below the lasing threshold for 250 ps during each 1-ns period to stop the light emission [see Fig. 3(a)]. The depletion of the light field forces the next lasing period to be triggered by the vacuum fluctuation and hence with random phase relative to the previous pulse [21]. The emission profile shows relaxation oscillations at the start of each pulse, which quickly settles into steady-state emission. Relaxation oscillations are caused by an initial overshoot when emission starts from a depleted cavity. The duration of each quasi-steady-state emission is long enough to coherently seed a pair of short output pulses. Adjacent short pulses seeded by different quasi-steady-state pulses will therefore have a random phase, as demonstrated in Fig. 3(b), whereas pulse pairs by the same seed pulse show a constant phase. Our source therefore meets necessary phase randomization for the security of the BB 84 protocol [13].

To fully demonstrate the suitability of the source for the BB84 protocol, we integrate the source in a BB84 transmitter and measure the resulting sifted key rate and quantum bit error rates (QBER's). The results are reported in Fig. 4(a). The experimental values (symbols) are in excellent agreement with the theoretical simulation (lines). The maximum transmission loss of about $40 \mathrm{~dB}$ (equivalent to $200 \mathrm{~km}$ of standard fiber) is limited by the detector noise. The QBER stays approximately constant at a base level of $2.4 \%$ for channel losses up to $30 \mathrm{~dB}$. This base value sets an upper bound for the encoding error of the light source as a BB84 encoder, which is comparable to the values achieved with conventional bulk or fiber optics [34].

Owing to its interferometer-free design, the phasemodulated source has excellent phase stability. This

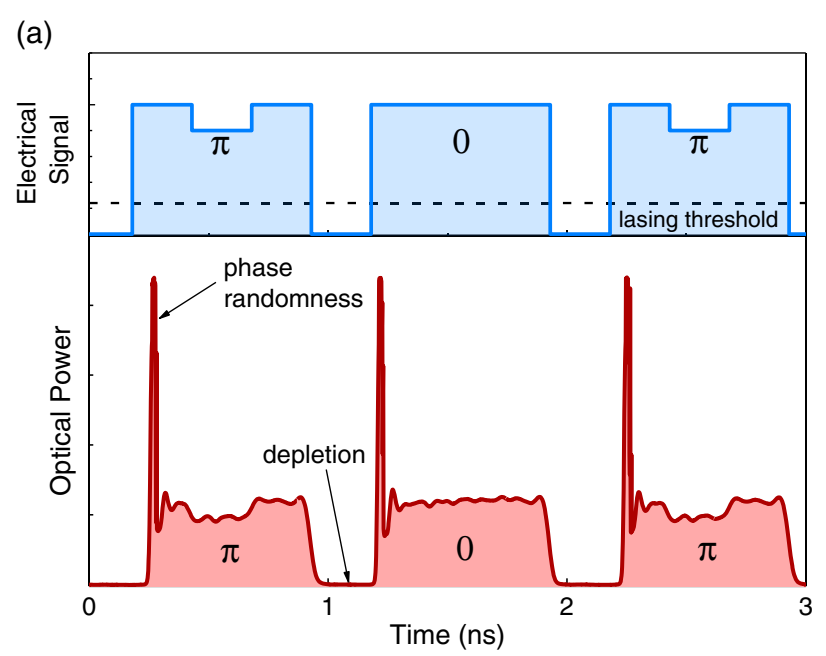

(b)

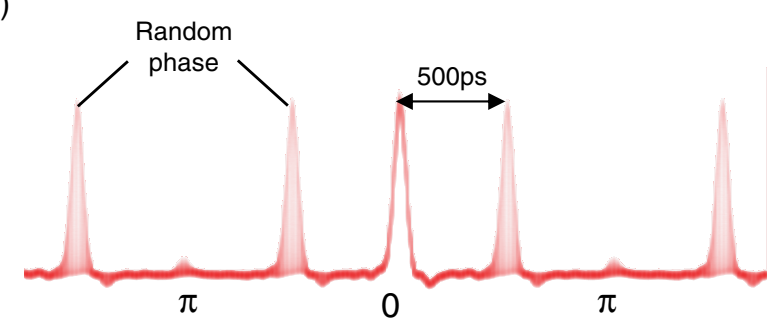

FIG. 3. Phase randomization pattern, adopted to guarantee security in the BB84 [13] and RR-DPS [33] protocol. We realize it here by depleting the cavity photon field in the phasepreparation laser prior between emission periods. (a) Upper panel: Driving signal applied to the phase-preparation laser. Lower panel: Temporal emission profile of the phase-preparation laser. (b) Color-coded density plot of the measured waveform of short pulses seeded by the injected light with the pattern shown in (a). The output with well-defined (random) intensity corresponds to the interference of short pulses seeded by the same (different) seeding pulse.

reduces the complexity of the QKD setup by removing the need for active stabilization of the phase. For illustration, we measure the QBER continuously over a 24-h period with no active feedback applied. The light source, and the receiving interferometer, are independently temperature controlled. The time-dependent QBER, sampled at an interval of $1 \mathrm{~s}$, fluctuates within a very narrow range around an average value of $2.41 \%$ with a standard deviation of $0.10 \%$ [see Fig. 4(c)]. We plot the distribution of the measured QBER in the inset, together with a simulation of the unavoidable shot-noise statistical fluctuation due to the finite count rate. The close resemblance between the two distributions suggests that the additional phase instability due to the light source is insignificant.

By simply applying different electrical signals, the light source can be reconfigured to accommodate a variety of QKD protocols, including DPS and its recent "roundrobin" variant (RR-DPS) [33]. To demonstrate this reconfigurability, we use the same BB84 optical setup to implement the DPS protocol. Figure 4(b) shows the 


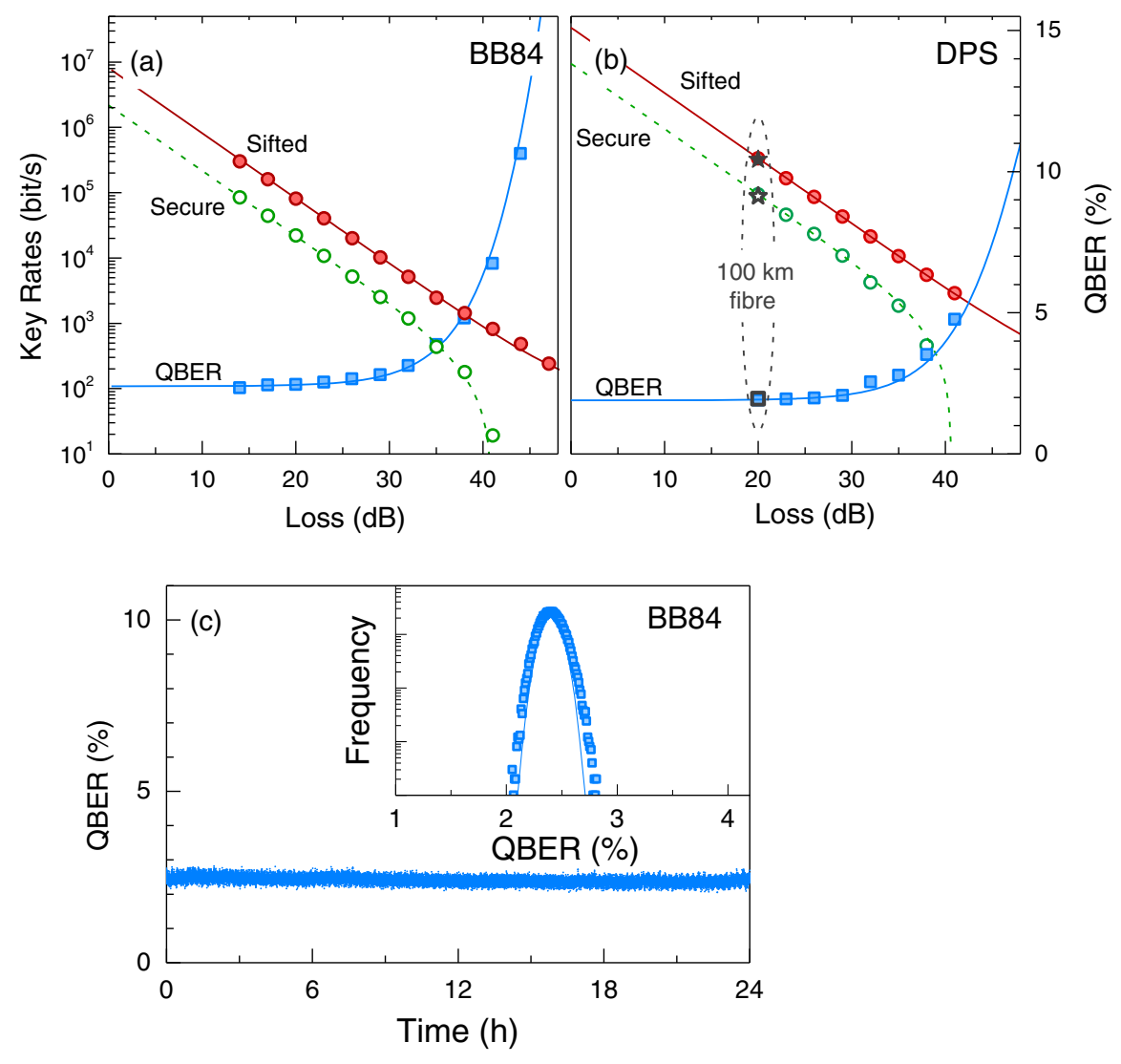

FIG. 4. QKD operating with the directly phase-modulated light source. Sifted and asymptotic secure rates and QBER versus channel loss for (a) BB84 and (b) DPS protocols. These QKD protocols use different security assumptions, and further details can be found in Appendix B. (c) QBER measured with the BB84 protocol for a duration of 24 hours. The inset shows the distribution of the measured QBER's (symbols) together with a simulation (line) of the shot-noise statistical fluctuation from the finite count rate. The signal integration time is $1 \mathrm{~s}$.

experimental results (symbols) together with the theoretical simulation (lines). The base QBER of $1.9 \%$ is well within the error threshold of the protocol. We also measure the performance over a $100-\mathrm{km}$ fiber spool, observing very similar error and bit rates to using the equivalent optical attenuation shown by the grey data points in the plot. It is also possible to implement the COW protocol by introducing a binary pattern in the driving signal to the pulse-generation laser.

\section{CONCLUSION}

In this work, we have demonstrated a novel directly modulated light source which exploits optical injection to prepare very pure phase states with exceptionally low driving voltage and which is suitable for challenging applications such as QKD. The phase-modulated source could be integrated into a fully functional phase transmitter with a size comparable to small-form pluggable (SFP) transceiver modules ubiquitously found in today's communication systems. Integration at this level is valuable and will find applications in heterogeneous networks where different quantum communication protocols coexist [23], or in access networks where the compactness and cost of the transmitters are of paramount importance [35]. Beyond communication applications, the direct source is useful to provide a stable phase-conditioned pulse sequence for the control of quantum systems, such as quantum dots [36] and parametric down-conversion [37].

\section{ACKNOWLEDGMENTS}

We thank J. Huwer for helping set up the superconducting detectors. G. L. R. acknowledges personal support via the EPSRC funded CDT in Photonic Systems Development and Toshiba Research Europe Limited.

\section{APPENDIX A: EXPERIMENTAL SETUP}

Fiber-pigtailed-distributed feedback laser diodes with built-in coolers are used for the setup shown in Fig. 1. The pulse-generation laser operates at room temperature. Under gain switching at $2 \mathrm{GHz}$, it is electrically biased just above its lasing threshold, producing an optical power of $25 \mu \mathrm{W}$ in the absence of optical injection. We temperature tune the wavelength of the phase-preparation laser for resonant 


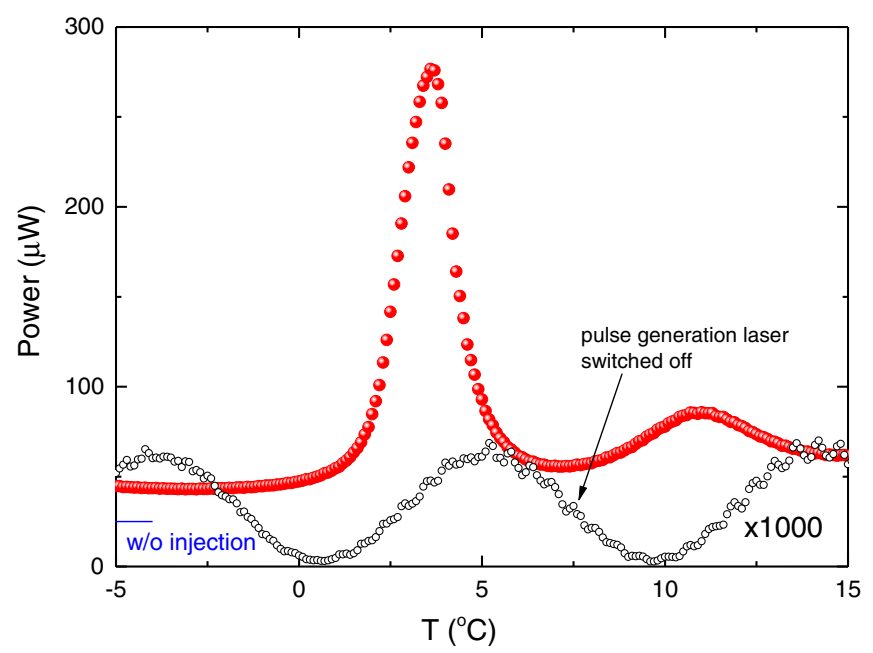

FIG. 5. Optical output power as a function of the operation temperature of the phase-preparation laser. The pulse-generation laser temperature is fixed, and the optical injection power is $50 \mu \mathrm{W}$. Also shown are the optical power in the absence of optical injection (blue line) and the optical reflection of the injected light measured with the pulse-generation laser switched off (open circles), multiplied by a factor of 1000 for clarity.

injection (see Fig. 5). Under the resonance, the output power increases by tenfold to $275 \mu \mathrm{W}$. The reflection of the phase-preparation laser light is found to be negligible, as evidenced in the measurement (open circles) with the pulse-generation laser switched off. In all experiments, we set the phase-preparation laser at the resonant temperature. The directly phase-modulated light source emits 70-ps pulses at $1551 \mathrm{~nm}$. Their temporal profile is shown in the leftmost panel of Fig. 2(a).

A planar-light-wave-circuit-based Mach-Zehnder interferometer [Fig. 2(a)] is used to evaluate the relative phase between adjacent pulses from the light source. The power splitting ratios of both input and output beam splitters are nominally $50 / 50$. The differential delay is $500 \mathrm{ps}$, and a built-in heater is used to adjust the interferometer phase. Under continuous-wave injection, we use an optical power meter to measure the interference visibility, which is found to increase with the injection power because of the increasing domination of the injected photons over the vacuum fluctuation in the injected cavity. The measured visibility starts to saturate at a modest injection power of $50 \mu \mathrm{W}$, and we obtain a maximum visibility of $99.02 \%$, which is limited by the coherence of the phase-preparation laser. Expectedly, we observe an increased visibility of 99.92\% when we replace the phase-preparation laser with a laser with longer coherence time (spectral width: $150 \mathrm{kHz}$ ).

To measure the data in Fig. 2(c), we apply a fixed modulation pattern to the phase-preparation laser to enable the direct source to produce a train of short pulses with a phase pattern containing 0 and $\Delta \phi$ phase modulations. Instead of using an optical power meter, we use an oscilloscope to record the interference results, which

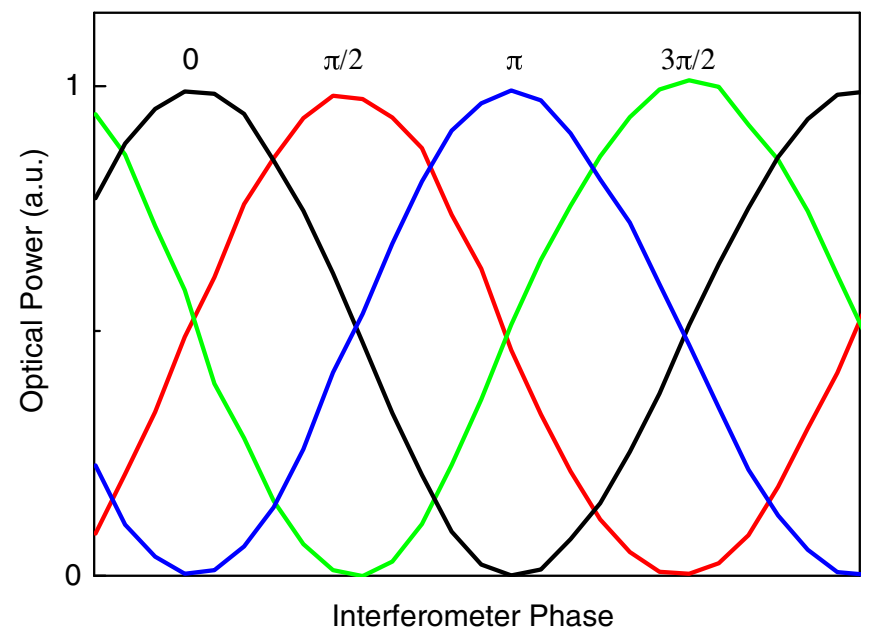

FIG. 6. Interference fringes recorded simultaneously for a repetitive modulation pattern of $0, \pi / 2, \pi$, and $3 \pi / 2$ phases.

are sorted according to their corresponding modulation values. By varying the interferometer phase, we obtain two separate sinusoidal interference fringes with a phase offset corresponding to $\Delta \phi$. For each modulation signal amplitude, we determine the $\Delta \phi$ value by comparing the interference fringes.

For illustration, we show in Fig. 6 the interference fringes recorded simultaneously for a repetitive modulation pattern of $0, \pi / 2, \pi$, and $3 \pi / 2$ phases.

\section{APPENDIX B: QUANTUM KEY DISTRIBUTION EXPERIMENTS}

The phase-modulated source transmits phase-encoded light pulses at a clock rate of $2 \mathrm{GHz}$, leading to effective QKD clock rates of 1 and $2 \mathrm{GHz}$ for the BB84 and DPS protocols, respectively. We use a quantum random number generator [21] to produce a 256-symbol sequence without intentional bias for each protocol. Modulation is applied to each pulse pair with a differential phase delay among $[0, \pi / 2, \pi$, and $3 \pi / 2]$ for the BB84 protocol, while each pulse is set to either the 0 or $\pi$ phase in relation to its preceding one in the DPS protocol. The corresponding modulation voltages are carefully calibrated to minimize the phase modulation error, which can have strong adverse effects on the final secure key rates [38,39]. The intensity of the source is heavily attenuated to the respective singlephoton levels, 0.5 and 0.4 photons/ns for the two protocols. An optical attenuator simulates the fiber channel with a loss scaling rate of $0.2 \mathrm{~dB} / \mathrm{km}$. Temperature-controlled planarlight-wave-circuit Mach-Zehnder interferometers of 3-dB loss are used for phase decoding, and the decoding basis is chosen passively with a 50/50 beam splitter for the BB84 protocol. For single-photon detection, we use a superconducting nanowire detector system featuring a detection efficiency of $13 \%-15 \%$ and a dark count rate of $150 \mathrm{~Hz}$ at a wavelength of $1550 \mathrm{~nm}$. Time-tagging electronics record 
photon detection events, from which we apply a 0.25 -ns detection window every $0.5 \mathrm{~ns}$ to extract sifted key rates and QBER's.

While the QBER's are the key parameter to measure the success of the light source as a QKD transmitter, we compute the secure key rates in the asymptotic scenario for the sake of completeness, following Refs. [40] and [41] for the decoy-state BB84 [42-44] and the DPS protocols, respectively. Although a finite-size effect [45] is not considered here, our light source is sufficiently stable, as shown in Fig. 4(c), to produce large data samples that are required for its mitigation. We note that the $\mathrm{QKD}$ protocols use different security assumptions. The decoy-state BB84 protocol is secure against the most general type of attacks, while the DPS protocol considers only individual attacks. For the decoy-state BB84 protocol, we choose 0.125 and 0 photons/ns for the intensities of the respective decoy and vacuum pulses.

[1] G.P. Agrawal, Fiber-Optic Communication Systems (John Wiley \& Sons, New York, 2002).

[2] I. Lucio-Martinez, P. Chan, X. Mo, S. Hosier, and W. Tittel, Proof-of-Concept of Real-World Quantum Key Distribution with Quantum Frames, New J. Phys. 11, 095001 (2009).

[3] P. J. Winzer and R. Essiambre, Advanced Optical Modulation Formats, Proc. IEEE 94, 952 (2006).

[4] B. E. A. Saleh and M. C. Teich, Fundamentals of Photonics (Wiley-Interscience, New York, 1991).

[5] K. Noguchi, O. Mitomi, and H. Miyazawa, Millimeter-Wave Ti:LiNbO ${ }_{3}$ Optical Modulators, J. Lightwave Technol. 16, 615 (1998).

[6] M. Smit et al., An Introduction to InP-Based Generic Integration Technology, Semicond. Sci. Technol. 29, 083001 (2014).

[7] R. Ding, T. Baehr-Jones, W. J. Kim, A. Spott, M. Fornier, J. M. Fedeli, S. Huang, J. Luo, A. K. Y. Jen, L. Dalton, and M. Hochberg, Sub-Volt Silicon-Organic Electro-optic Modulator with $500 \mathrm{MHz}$ Bandwidth, J. Lightwave Technol. 29, 1112 (2011).

[8] Y. Shi, C. Zhang, H. Zhang, J. H. Bechtel, L. R. Dalton, B. H. Robinson, and W. H. Steier, Low (sub-1-volt) Halfwave Voltage Polymeric Electro-optic Modulators Achieved by Controlling Chromophore Shape, Science 288, 119 (2000).

[9] Y. Enami et al., Hybrid Polymer/Sol-Gel Waveguide Modulators with Exceptionally Large Electro-Optic Coefficients, Nat. Photonics 1, 180 (2007).

[10] R. S. Tucker, High-Speed Modulation of Semiconductor Lasers, IEEE Trans. Electron Devices 32, 2572 (1985).

[11] T. Yamamoto, High-Speed Directly Modulated Lasers, in Optical Fiber Communication Conference (Optical Society of America, Los Angeles, 2012), p. OTh3F.5.

[12] I. N. Cano, A. Lerin, V. Polo, and J. Prat, Direct Phase Modulation DFBs for Cost-Effective ONU Transmitter in udWDM PONs, IEEE Photonics Technol. Lett. 26, 973 (2014).
[13] C. H. Bennett and G. Brassard, in Proceedings of the IEEE International Conference on Computers, Systems and Signal Processing, Bangalore, India, 1984 (IEEE, New York, 1984), pp. 175-179.

[14] N. Gisin, G. Ribordy, W. Tittel, and H. Zbinden, Quantum Cryptography, Rev. Mod. Phys. 74, 145 (2002).

[15] H. K. Lo, M. Curty, and K. Tamaki, Secure Quantum Key Distribution, Nat. Photonics 8, 595 (2014).

[16] V. Scarani and C. Kurtsiefer, The Black Paper of Quantum Cryptography: Real Implementation Problems, Theor. Comput. Sci. 560, 27 (2014).

[17] G. H. M. van Tartwijk and D. Lenstra, Semiconductor Lasers with Optical Injection and Feedback, Quantum Semiclass. Opt. B 7, 87 (1995).

[18] E. K. Lau, L. J. Wong, and M. C. Wu, Enhanced Modulation Characteristics of Optical Injection-Locked Lasers: A Tutorial, IEEE J. Sel. Top. Quantum Electron. 15, 618 (2009).

[19] L. C. Comandar, M. Lucamarini, B. Fröhlich, J. F. Dynes, A. W. Sharpe, S. W. B. Tam, Z. L. Yuan, R. V. Penty, and A.J. Shields, Quantum Key Distribution without Detector Vulnerabilities Using Optically Seeded Lasers, Nat. Photonics 10, 312 (2016).

[20] B. R. Bennett, R. A. Soref, and J. A. del Alamo, CarrierInduced Change in Refractive Index of InP, GaAs and InGaAsP, IEEE J. Quantum Electron. 26, 113 (1990).

[21] Z. L. Yuan, M. Lucamarini, J. F. Dynes, B. Fröhlich, A. Plews, and A.J. Shields, Robust Random Number Generation Using Steady-State Emission of GainSwitched Laser Diodes, Appl. Phys. Lett. 104, 261112 (2014).

[22] M. Peev et al., The SECOQC Quantum Key Distribution Network in Vienna, New J. Phys. 11, 075001 (2009).

[23] M. Sasaki et al., Field Test of Quantum Key Distribution in the Tokyo QKD Network, Opt. Express 19, 10387 (2011).

[24] S. Wang et al., Field and Long-Term Demonstration of a Wide Area Quantum Key Distribution Network, Opt. Express 22, 21739 (2014).

[25] Y.L. Tang et al., Measurement-Device-Independent Quantum Key Distribution over Untrustful Metropolitan Network, Phys. Rev. X 6, 011024 (2016).

[26] D. Gottesman, H.-K. Lo, N. Lütkenhaus, and J. Preskill, Security of Quantum Key Distribution with Imperfect Devices, Quantum Inf. Comput. 4, 325 (2004).

[27] C. H. Bennett, Quantum Cryptography Using Any Two Nonorthogonal States, Phys. Rev. Lett. 68, 3121 (1992).

[28] P. D. Townsend, Secure Key Distribution System Based on Quantum Cryptography, Electron. Lett. 30, 809 (1994).

[29] R. J. Hughes, G. L. Morgan, and C. G. Peterson, Quantum Key Distribution over a $48 \mathrm{~km}$ Optical Fibre Network, J. Mod. Opt. 47, 533 (2000).

[30] Z. L. Yuan and A. J. Shields, Continuous Operation of a One-Way Quantum Key Distribution System over Installed Telecom Fibre, Opt. Express 13, 660 (2005).

[31] K. Inoue, E. Waks, and Y. Yamamoto, Differential Phase Shift Quantum Key Distribution, Phys. Rev. Lett. 89, 037902 (2002).

[32] D. Stucki, N. Brunner, N. Gisin, V. Scarani, and H. Zbinden, Fast and Simple One-Way Quantum Key Distribution, Appl. Phys. Lett. 87, 194108 (2005). 
[33] T. Sasaki, Y. Yamamoto, and M. Koashi, Practical Quantum Key Distribution Protocol without Monitoring Signal Disturbance, Nature (London) 509, 475 (2014).

[34] M. Lucamarini, K. A. Patel, J. F. Dynes, B. Fröhlich, A. W. Sharpe, A. R. Dixon, Z. L. Yuan, R. V. Penty, and A. J. Shields, Efficient Decoy-State Quantum Key Distribution with Quantified Security, Opt. Express 21, 24550 (2013).

[35] B. Fröhlich, J. F. Dynes, M. Lucamarini, A. W. Sharpe, Z. L. Yuan, and A. J. Shields, A Quantum Access Network, Nature (London) 501, 69 (2013).

[36] H. Jayakumar, A. Predojević, T. Kauten, T. Huber, G. S. Solomon, and G. Weihs, Time-Bin Entangled Photons from a Quantum Dot, Nat. Commun. 5, 4251 (2014).

[37] H. De Riedmatten, I. Marcikic, H. Zbinden, and N. Gisin, Creating High Dimensional Entanglement Using ModeLocked Lasers, Quantum Inf. Comput. 2, 425 (2002).

[38] K. Tamaki, M. Curty, G. Kato, H.-K. Lo, and K. Azuma, Loss-Tolerant Quantum Cryptography with Imperfect Sources, Phys. Rev. A 90, 052314 (2014).

[39] F. Xu, K. Wei, S. Sajeed, S. Kaiser, S. Sun, Z. Tang, L. Qian, V. Makarov, and H.-K. Lo, Experimental Quantum Key
Distribution with Source Flaws, Phys. Rev. A 92, 032305 (2015).

[40] X. F. Ma, B. Qi, Y. Zhao, and H.-K. Lo, Practical Decoy State for Quantum Key Distribution, Phys. Rev. A 72, 012326 (2005).

[41] E. Waks, H. Takesue, and Y. Yamamoto, Security of Differential-Phase-Shift Quantum Key Distribution Against Individual Attacks, Phys. Rev. A 73, 012344 (2006).

[42] W. Y. Hwang, Quantum Key Distribution with High Loss: Toward Global Secure Communication, Phys. Rev. Lett. 91, 057901 (2003).

[43] H. K. Lo, X. F. Ma, and K. Chen, Decoy State Quantum Key Distribution, Phys. Rev. Lett. 94, 230504 (2005).

[44] X. B. Wang, Beating the Photon-Number-Splitting Attack in Practical Quantum Cryptography, Phys. Rev. Lett. 94, 230503 (2005).

[45] V. Scarani and R. Renner, Quantum Cryptography with Finite Resources: Unconditional Security Bound for Discrete-Variable Protocols with One-Way Postprocessing, Phys. Rev. Lett. 100, 200501 (2008). 